'Programa Doctorado en Enfermería Universidad de

Concepción. Concepción, Chile.

2Departamento del Adulto y

Adulto Mayor. Facultad de

Enfermería. Universidad de

Concepción. Concepción, Chile.

${ }^{3}$ Sección de Nefrología, Hospital

del Salvador, Departamento de

Medicina Oriente, Universidad de

Chile. Santiago, Chile.

aEnfermera.

bPhd().

'Phd.

Trabajo no recibió financiamiento externo.

Los autores declaran no tener conflictos de interés.

Recibido el 17 de noviembre de 2018, aceptado el 15 de marzo de 2019.

Correspondencia a: Rosa Alcayaga D. Facultad de Enfermería,

Universidad de Concepción. Víctor Lamas 1135, Concepción, Región del Bío Bío. Concepción, Chile. ralcayaga@udec.cl

\section{Diseño de una propuesta de protocolo de estandarización y gestión de calidad para el proceso de procuramiento de órganos}

\author{
ROSA ALCAYAGA DROGUETT ${ }^{1, \mathrm{a}, \mathrm{b}}$, JASNA STIEPOVICH BERTONI ${ }^{2, \mathrm{a}}$, \\ FERNANDO GONZÁLEZ FUENZALIDA ${ }^{3, c}$
}

\section{Proposal of a management and standardiza- tion protocol for organ procurement in Chile}

Background: Organ donation rates for transplantation are low in Chile and there is a paucity of a technical-structured-normalized procurement process. Aim: To design a protocol to standardize the organ procurement process based on the Chilean reality. Material and Methods: After a first phase of bibliographic reviews and analyzing de Chilean legislative and normative frame, a proposal was elaborated in collaboration with five expert coordinators. In the second phase, two Delphi rounds with the local coordinators of the organ procurement centers were carried out. Their responses were analyzed and the stages and criteria to standardize the organ procurement process were determined. Results: The expert defined organ procurement process, validated by local coordinators, comprised nine stages and 36 criteria. The expert opinion coincidence was high and significant (Cronbach's alpha $>0.8, p<0.01$ ). Conclusions: The protocol describing the procurement process, constructed and validated by experts, will allow to standardize a clinical protocol contributing to a successful national organ procurement.

(Rev Med Chile 2019; 147: 296-304)

Key words: Organ Transplantation; Quality of Health Care; Tissue and Organ Procurement; Transplantation.

\section{L} os trasplantes de órganos se han convertido en una solución para resolver la condición de personas que, de no tenerlo, ven limitada su salud y, muchas veces, su vida ${ }^{1}$. Pero, para poder realizar trasplantes de donante no vivo, se deben obtener estos órganos en las mejores condiciones posibles, mediante un protocolo clínico de procuramiento de órganos ${ }^{2}$, el cual ha sido definido como: "el proceso mediante el cual, y a través de la ejecución de etapas bien definidas, se logra que los órganos de alguien que ha fallecido, sean implantados en un receptor compatible" 3 .

España es referente mundial en esta materia, logrando tasas de donación de 43,8 donantes por millón de habitantes (pmp), cifra que supera con creces la tasa de 7,4 donantes pmp alcanzada por Chile en el año 2016 ${ }^{4}$. La Organización Nacional de Trasplantes de España (ONT) ${ }^{5}$, es la institución responsable de implementar y controlar que los procesos de procuramiento se lleven a cabo correctamente ${ }^{6}$. En este modelo español, es fundamental el rol que juega el Coordinador de trasplantes, quien es un profesional sanitario encargado desde la detección, selección y mantenimiento del posible donante, hasta la logística de extracción y distribución de órganos y tejidos?

En Chile, este proceso es dependiente del Ministerio de Salud, a través de la Coordinadora 
Nacional de Procuramiento y Trasplante (CNTP) dependiente de la Subsecretaría de Redes Asistenciales, esta CNTP es responsable de generar donantes efectivos en todos los centros asistenciales públicos y privados que tengan capacidad para hacerlo, las coordinaciones locales de procuramiento (CLP) se componen principalmente por enfermeras/os, quienes son responsables de llevar a cabo el programa de procuramiento de órganos ${ }^{8}$.

Como una forma de optimizar este proceso, el Minsal, en el año 2015, elaboró el documento "Orientaciones Técnicas del Proceso de Procuramiento de Órganos y Tejidos para Trasplante", del cual surgen recomendaciones para el funcionamiento de los programas de procuramiento de órganos a nivel nacional ${ }^{9}$. Además desde el año 2011, se implementa el programa informático SIDOT (Sistema Integrado de Donación y Trasplantes) que registra la información de los potenciales donantes a nivel nacional, permitiendo un seguimiento diario en línea de la evolución del proceso, pero no asegura una donación efectiva.

Estas orientaciones técnicas, si bien no corresponden a una normativa, describen la Organización y las funciones de la Coordinadora Nacional del Minsal. Los procesos de donación, deben ser desarrollados basados en estas Orientaciones, que establecen 5 etapas asociadas al procuramiento: a) Detección, evaluación y seguimiento de posibles donantes; b) Mantención de todos los pacientes con potencialidad de donación; c) Certificación de muerte encefálica de potenciales donantes, d) Solicitud de testimonio de última voluntad, e) Procedimiento de extracción de órganos y tejidos y trasplante?

Sin embargo, a nivel nacional se observa una baja sostenida de donantes (de 13 a 7,4 donantes pmp en los últimos 5 años), y una alta y sostenida negativa familiar $(51 \%)^{10,11}$. Frente a este escenario, es necesario identificar los nodos críticos que pudieran estar dificultando la donación efectiva. En Chile el proceso de obtención de donantes de órganos para trasplante al no ser estandarizado, carece de puntos objetivos de referencia contra los cuales validar las prácticas locales de los centros de procuramiento.

Este estudio se propuso cómo objetivo elaborar un protocolo del proceso de procuramiento de órganos en Chile, para posteriormente constituirse en una propuesta para la estandarización y gestión de calidad del proceso de procuramiento de órganos.

\section{Metodología}

Para la estandarización del proceso de procuramiento se desarrolló la siguiente metodología:

1) Revisión de la literatura biomédica y Orientaciones Técnicas del Minsal, para tener una primera aproximación al proceso de procuramiento en Chile e identificar buenas prácticas en el tema ${ }^{12-17}$.

2) Entrevistas grupales y reuniones de acuerdos con cinco enfermeros coordinadores de procuramiento de distintos centros hospitalarios como expertos, de ellos, tres son Coordinadoras Locales de Hospitales públicos de gran complejidad, dos del área Metropolitana y uno de la V Región, con 20, 8 y 10 años de experiencia como coordinador local , un enfermero Coordinador central de Procuramiento de órganos del Minsal, con 6 años de experiencia en el cargo y una enfermera encargada del programa de Procuramiento de órganos de la Coordinadora Nacional de Procuramiento del Minsal con 3 años de experiencia a cargo de la coordinación nacional.

En las entrevistas grupales, los temas emergentes de la revisión bibliográfica fueron discutidos en conjunto con los 5 participantes, se reordenaron, clasificaron y se describieron en frases con sentido de acción, para definir propuesta de las etapas y criterios del Protocolo de Procuramiento. En esta fase, se identificaron 9 etapas del proceso de procuramiento de órganos y 39 criterios, que fueron plenamente consensuados en el grupo.

3) Aplicación de la Técnica Delphi para recolectar sistemáticamente opiniones de los coordinadores locales a nivel nacional y construir consenso de grupo, a partir del procesamiento estadístico de las diferencias y coincidencias entre las apreciaciones individuales, efectuándose en este estudio 2 rondas. Se destaca que en la Técnica Delphi, cada experto realiza la evaluación y se recogen las opiniones de manera anónima ${ }^{18}$.

4) En Delphi I participaron 42 enfermeros coordinadores de procuramiento locales de los centros hospitalarios, públicos y privados, donde se realiza búsqueda de potenciales donantes de órganos. Fueron invitados 58 coordinadores a participar del Delphi I, 42 de ellos manifestaron aceptación (72\%). Con el apoyo de la Coordina- 
dora Nacional del Minsal, se les envió un mail con información del proyecto, además se adjuntó el consentimiento informado y la encuesta Delphi I. Los coordinadores que aceptaron, firmaron un consentimiento informado aprobado por el Comité de Ética de la Universidad de Concepción y respondieron en fecha convenida (dos semanas). Este proceso fue efectuado en el caso del Delphi I hasta mayo de 2018 y el Delphi II hasta julio de 2018.

La primera encuesta Delphi I, constaba 9 etapas del proceso de procuramiento y 39 criterios identificados en fase precedente de la construcción de la encuesta y debía ser respondida con un margen de consenso como Acuerdo o Desacuerdo y otorgarles un grado de importancia individual en una escala de Likert de 1 a 7 , según tuvieran muy escasa o nula importancia hasta muy alta o extrema importancia, respectivamente. Además, se les ofreció la opción de indicar observaciones por cada etapa (datos cualitativos).

Con base a las respuestas de la primera encuesta se realizó una categorización de comentarios cualitativos del Delphi I, análisis de las respuestas, una validación de cada etapa y de los criterios definidos por etapas del proceso procuramiento por parte del panel de 5 expertos y se incorporan a la segunda encuesta a remitir, Delphi II. Se envió a los 42 coordinadores locales un documento que condensaba por ítem los resultados de la primera encuesta. Allí se les expresaba lo que se había obtenido en margen de acuerdo y del nivel de importancia por ítem, además se planteaba lo que debían evaluar en la segunda ronda, Delphi II, de estos, se recepcionaron 35 respuestas (83\%).

5) Análisis de datos: Las respuestas individuales por pregunta de las encuestas Delphi I y Delphi II fueron agrupadas y analizadas con el test de Kuder Richardson 20 para las respuestas binarias (acuerdo o desacuerdo) y alfa de Cronbach, para estudiar la consistencia interna de las respuestas con siete categorías de Likert (1-7), y test de correlación por rangos de Spearman utilizando IBM SPSS software versión 20,0 for Windows (IBM, Armonk, NY, USA).

\section{Resultados}

Las respuestas del Delphi I sobre las 9 etapas, fueron bastante semejantes entre los expertos, de hecho, concordaron completamente en 7 etapas (varianza 0 ) y en las etapas 5 y 9 , las concordancias tuvieron medias y (varianzas) de $0,919(0,075)$ y $0,963(0,36)$, respectivamente, pero sólo la primera fue estadísticamente significativa $(\mathrm{p}=0,037)$. Por tanto, se mantienen las 9 etapas propuestas del proceso de procuramiento.

Respecto del grado de importancia de cada uno de los criterios, componentes de cada etapa, las respuestas se muestran en (Tabla 1). Se seleccionaron aquellos criterios que concitaban más de $80 \%$ de acuerdo, de los 39 criterios 3 de ellos fueron eliminados, estos corresponden uno de la tercera etapa: Valoración y Mantención del Potencial Donante, el criterio 3.6 Registro en un documento formal de los eventos durante el seguimiento de mantenimiento y dos criterios de la quinta etapa Solicitud de Última Voluntad: criterio 5.4 Registro de cambio de potenciales donantes a donantes efectivos y criterio 5.5 Existencia de Protocolo de Paro Cardíaco repentino del donante. Los restantes 36 criterios se mantuvieron para ser parte de la segunda encuesta (Delphi II).

Los resultados del Delphi II mostraron perfecta concordancia entre las respuestas de los 35 coordinadores en las 9 etapas (varianza 0 ). Sin embargo, las concordancias en la importancia dada a cada criterio variaron. Para la determinación de los resultados, como se establece la relación y consistencia de las respuestas, entre la primera ronda (Delphi I) y segunda ronda (Delphi II), se procedió según lo explicitado en (Tabla 2).

El alfa de Cronbach del Delphi I fue de 0,623; $\mathrm{p}<0,001$ y el Alfa de Cronbach del Delphi II fue de $0,801, p<0,001$. Este último valor es considerado como ilustrativo de un alto y significativo consenso entre los expertos.

Las 9 etapas del Proceso de Procuramiento resultaron ser:

\section{a) Etapa 1}

Detección y Selección del Posible Donante, es la primera y más importante etapa de este proceso y comienza con la pesquisa de los posibles donantes, siendo el primer eslabón de la cadena donación-trasplante, compuesta por una serie de actividades clínico-asistenciales y administrativas que permiten concluir con la extracción de órganos para trasplante. Los criterios propuestos para esta etapa: 1.5 Protocolo formal de detección de posibles donantes, 1.10 Capacitación periódica de detección y selección de donante y 1.12 Pesquisar 
Tabla 1. Criterios iniciales por etapas del DELPHI I

\begin{tabular}{|c|c|c|c|}
\hline & Media & $\begin{array}{l}\text { D } \\
\text { estándar }\end{array}$ & $\begin{array}{c}\% \\
\text { acuerdo }\end{array}$ \\
\hline \multicolumn{4}{|l|}{ Etapa 1: Detección y Selección del Posible Donante } \\
\hline 1.1. Mantiene Documentos de Autorización a la donación & 7 & 0 & $100 \%$ \\
\hline 1.2. Mantiene Manual actualizado de procedimientos en Procuramiento & 6,97 & 0,15 & $100 \%$ \\
\hline 1.3. Espacio físico exclusivo como Oficina de Procuramiento & 6,97 & 0,15 & $100 \%$ \\
\hline 1.4. Computador operativo y uso exclusivo & 6,90 & 0,49 & $100 \%$ \\
\hline 1.5. Mantiene Protocolo de detección posibles donantes & 6,63 & 0,53 & $100 \%$ \\
\hline 1.6. Cargo de coordinador local a tiempo completo & 7 & 0 & $100 \%$ \\
\hline 1.7. Respeta tiempos dedicación exclusiva al cargo & 6,95 & 0,21 & $100 \%$ \\
\hline 1.8. Mantiene cobertura de turnos de Coordinador $24 / 7$ & 7 & 0 & $100 \%$ \\
\hline 1.9. Comparte cargo con dos o más coordinadores & 7 & 0 & $100 \%$ \\
\hline 1.10. Programa de Capacitación periódica de detección de Donante & 6,41 & 0,74 & $100 \%$ \\
\hline 1.11. Notificación de posible y luego potencial donante a Coordinación Central & 6,53 & 0,63 & $100 \%$ \\
\hline $\begin{array}{l}\text { 1.12. Pesquisa diariamente a todos los pacientes ingresados a Urgencias o UCl } \\
\text { con Glasgow-7 y lesión cerebral aguda }\end{array}$ & 6,61 & 0,89 & $100 \%$ \\
\hline \multicolumn{4}{|l|}{ Etapa 2: Ingreso a Seguimiento } \\
\hline 2.1. Realiza visita diaria de seguimiento del posible donante & 6,87 & 0,4 & $100 \%$ \\
\hline 2.2. Registra datos, exámenes del posible donante en SIDOT & 6,78 & 0,69 & $100 \%$ \\
\hline \multicolumn{4}{|l|}{ Etapa 3: Valoración y Mantención del Potencial donante } \\
\hline 3.1. Mantiene Protocolo formal de mantención del potencial donante & 6,85 & 0,35 & $100 \%$ \\
\hline 3.2. Visita diaria seguimiento del Coordinador Local, ( tiempo asignado) & 6,61 & 0,83 & $100 \%$ \\
\hline 3.3. Registro en ficha clínica, del médico a cargo de la mantención & 6,92 & 0,07 & $88 \%$ \\
\hline 3.4. Disponibilidad de Cama en UCl y VM, para potencial donante & 7 & 0 & $100 \%$ \\
\hline 3.5. Cumplimiento de Protocolo durante la fase de mantención & 7 & 0 & $100 \%$ \\
\hline $\begin{array}{l}\text { 3.6. Registro en documento formal de eventos adversos durante el seguimiento } \\
\text { de mantenimiento }\end{array}$ & 6,29 & 1,14 & $76,1 \%$ \\
\hline \multicolumn{4}{|l|}{ Etapa 4: Certificación Muerte Encefálica } \\
\hline 4.1. Evaluación de donante en muerte encefálica por Neurólogo o Neurocirujano & 7 & 0 & $100 \%$ \\
\hline 4.2. Tecnólogo Médico de llamada, para toma EEG & 6,85 & 0,35 & $100 \%$ \\
\hline 4.3. Equipo de EEG operativo y disponible $24 \mathrm{~h}$ & 6,85 & 0,47 & $90 \%$ \\
\hline 4.4. Neurocirujano o Neurólogo de turno o de llamada, certificar Muerte Encefálica & 7 & 0 & $100 \%$ \\
\hline 4.5. Disponibilidad de TAC cerebro, $24 \mathrm{~h}$ & 6,82 & 0,62 & $88 \%$ \\
\hline \multicolumn{4}{|l|}{ Etapa 5: Solicitud de Última Voluntad (STUV) } \\
\hline 5.1. Lugar físico, para realizar solicitar donación & 7 & 0 & $100 \%$ \\
\hline 5.2. Entrevista de STUV según protocolo establecido & 6,85 & 0,52 & $97 \%$ \\
\hline 5.3. Registro local de causa de negativa familiar & 6,73 & 0,97 & $100 \%$ \\
\hline 5.4. Registro de cambio de potenciales donantes a donantes efectivos & 6,02 & 1,79 & $69 \%$ \\
\hline 5.5. Existencia de Protocolo de Paro Cardíaco repentino del donante & 6,31 & 1,61 & $76,1 \%$ \\
\hline \multicolumn{4}{|l|}{ Etapa 6: Selección de Receptores } \\
\hline 6.1. Comunicación con Coordinador Central, según protocolo & 7 & 0 & $100 \%$ \\
\hline 6.2. Protocolo de acuerdo con equipos extractores, tiempos de respuestas & 6,85 & 0,47 & $98 \%$ \\
\hline 6.3. Comunicación efectiva con los equipos quirúrgicos para extracción & 7 & 0 & $100 \%$ \\
\hline \multicolumn{4}{|l|}{ Etapa 7: Coordinación Pabellón y extracción } \\
\hline 7.1. Protocolos de registro de datos de etapas del proceso de extracción. & 6,97 & 0,15 & $100 \%$ \\
\hline 7.2. Protocolo de registro de causa de descarte de órganos (intra pabellón) & 6,80 & 0,45 & $100 \%$ \\
\hline \multicolumn{4}{|l|}{ Etapa 8: Preservación y Traslado } \\
\hline 8.1. Protocolo de registro de los órganos donados y su asignación & 6,80 & 0,45 & $100 \%$ \\
\hline 8.2. Verificación de recepción de órganos en condiciones de ser implantados & 6,85 & 0,42 & $100 \%$ \\
\hline \multicolumn{4}{|l|}{ Etapa 9: Entrega del cuerpo } \\
\hline 9.1. Protocolo de registro de entrega del cuerpo a anatomía patológica o médico legal & 6,97 & 0,15 & $92,8 \%$ \\
\hline 9.2. Protocolo de entrega del cuerpo a la familia & 6,78 & 0,96 & $97,6 \%$ \\
\hline
\end{tabular}




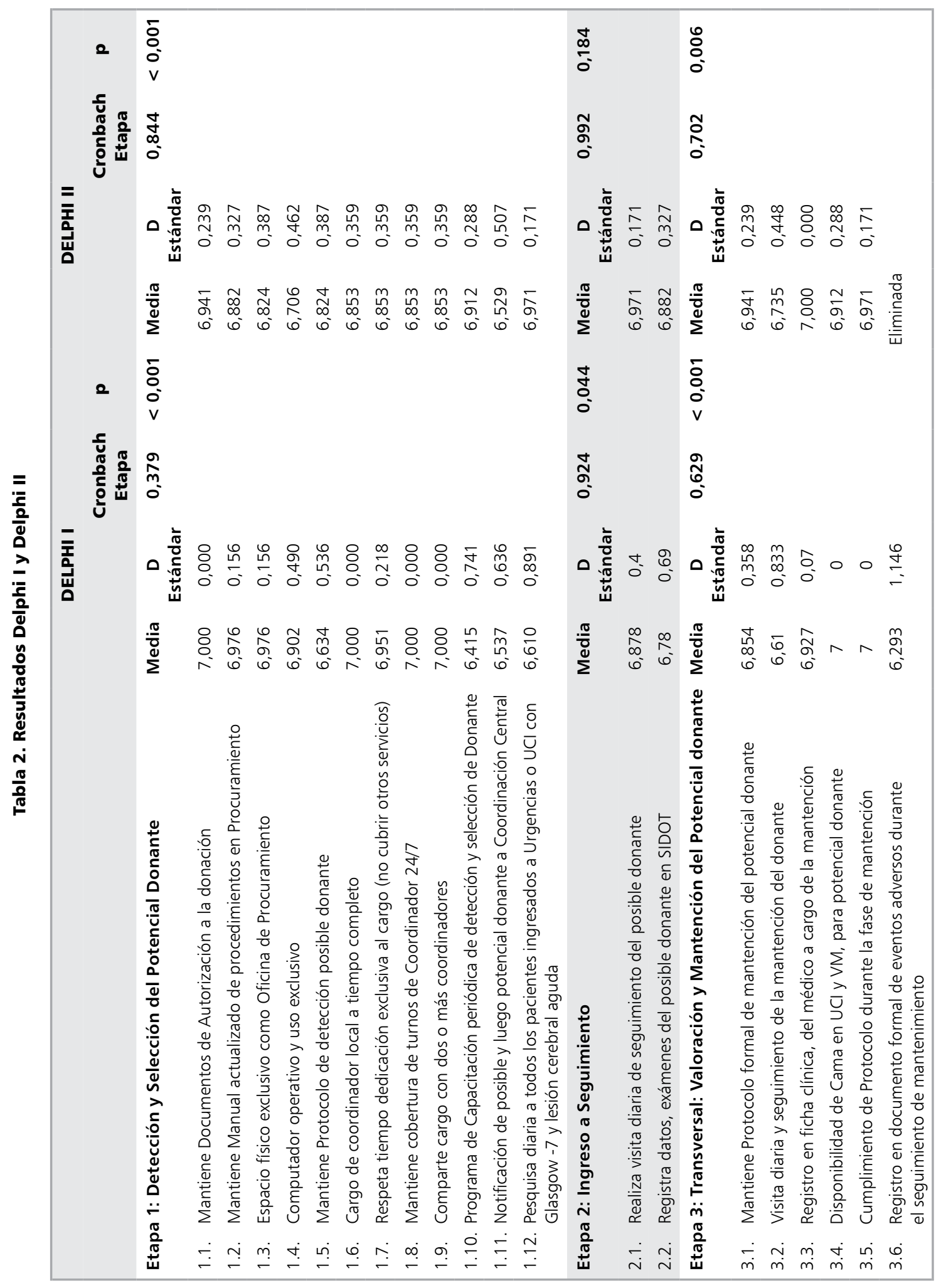




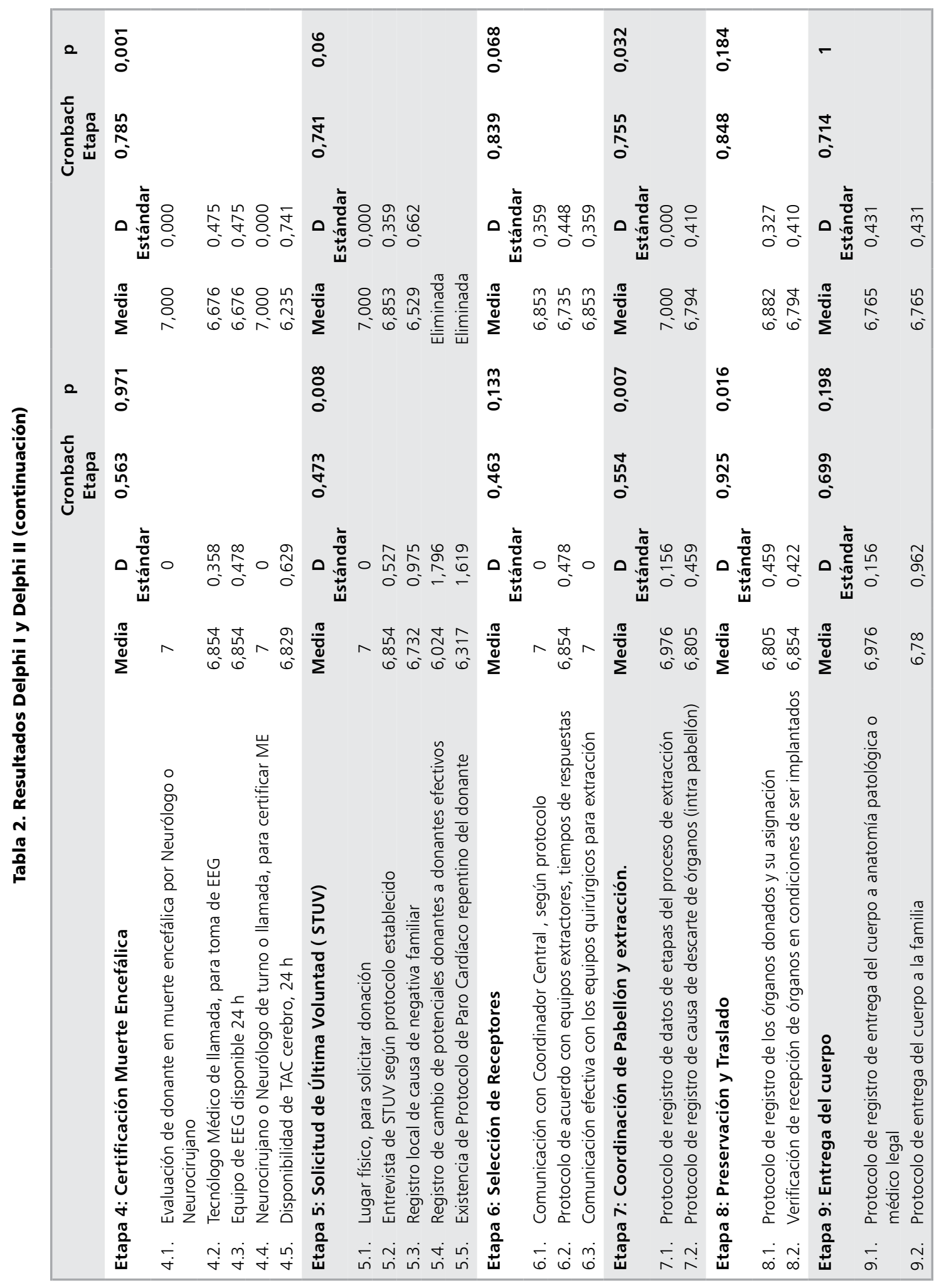


a todos los pacientes ingresados a Urgencia o UCI con Glasgow $<7$, se evidencian como menos importantes en el Delphi I, logrando acuerdo en el Delphi II.

\section{b) Etapa 2}

Ingreso a Seguimiento, en esta etapa, el criterio 2.1 realizar visita diaria al posible donante y criterio 2.2 contar con registro diario de la evolución, para realizar un seguimiento acabado del posible donante, logran consenso en ambas rondas de consulta.

\section{c) Etapa 3}

Valoración y Mantención del Potencial donante, esta etapa es transversal a todo el proceso de procuramiento, es un proceso activo, con múltiples factores involucrados del potencial donante de órganos en condiciones de perfusión y oxigenación óptimas, que aseguren una correcta viabilidad de los órganos para ser trasplantados. El consenso logrado en los criterios 3.4: Disponibilidad de Cama en UCI y VM, para potencial donante y 3.5: Cumplimiento de Protocolo durante la fase de mantención en las 6 horas previas a la extracción de órganos, valida ambos criterios como muy importantes.

\section{d) Etapa 4}

Certificación de Muerte Encefálica, etapa donde el donante pasa de posible donante a potencial donante. No se considera importante criterio 4.5 Disponer de TAC cerebro, 24 horas, sí se considera de vital importancia el criterio 4.3. Disponer de Neurocirujano o Neurólogo de turno o de llamada, para certificar Muerte Encefálica, ya que no en todos los establecimientos hospitalarios se cuenta con este especialista.

\section{e) Etapa 5}

Solicitud de Última Voluntad, solicitud a la familia de la donación de órganos, etapa decisiva, ya que, de haber negativa, se detiene el proceso de procuramiento. No existe consenso en criterio 5.3 registros de causa de negativa familiar.

\section{f) Etapa 6}

Selección de Receptores, a quienes irán destinados los órganos donados, si bien en esta etapa interviene principalmente el Coordinador central, el criterio 6.2 Protocolo de acuerdo con los equi- pos extractores en cuanto a respetar tiempos de coordinación, no logra consenso.

\section{g) Etapa 7}

Coordinación Pabellón y Extracción, el criterio 7.2 Protocolo de registro de causa de descarte de órganos y/o eventos adversos (intra pabellón) logra consenso, respecto a registrar los eventos adversos ocurridos durante la extracción.

\section{h) Etapa 8}

Preservación y Traslado de los Órganos, es crucial entregar órganos bien embalados para su conservación, el criterio 8.1 Protocolo registro de órganos donados, logra conceso como importante entre los coordinadores.

\section{i) Etapa 9}

Entrega del Cuerpo del donante en óptimas condiciones, por ética y respeto a la familia que autorizó la donación, criterio 9.2 Protocolo de entrega del cuerpo a la familia, no logra consenso entre las respuestas.

Por tanto, las 9 etapas del proceso de procuramiento y los 36 criterios que conforman la propuesta del protocolo de estandarización se encuentran explicitados en Tabla 2 del presente documento.

\section{Discusión}

En un proceso como el procuramiento de órganos, todos y cada uno de sus eslabones debe ser suficientemente sólido como para garantizar que la cadena no se rompa. Llama la atención respecto a tres etapas donde sus respectivos criterios tuvieron bajo acuerdo en el Delphi I, pero que logran consenso en el Delphi II.

- Etapa 1: Detección y selección del potencial donante. Los coordinadores locales concuerdan en que es necesario contar con elementos administrativos y de ordenamiento de gestión que les permitan desempeñarse permanentemente en turnos de procuramiento, no cumpliendo otras funciones.

- Etapa 4: Certificación de Muerte Encefálica. Existe desacuerdo, aun cuando la ley de donación de órganos establece claramente que es imprescindible el contar con estudios ana- 
tómicos del cerebro como, tomografía axial, y no así de electroencefalografía, a excepción de pacientes pediátricos. Esto, supone que el conocimiento del marco jurídico que regula la actividad de procuramiento no es del dominio transversal de todos los coordinadores.

- Etapa 5: Solicitud de Última Voluntad: Esta etapa es del todo crucial en el proceso de donación, tanto, que explica que de los potenciales donantes que llegan a ella, más del $50 \%$ de los familiares, no consienten en la donación. Al observar las respuestas de los coordinadores, da la impresión que el enfrentamiento de esta etapa dista de ser estandarizada.

En consecuencia, pese a las Orientaciones Técnicas del Minsal, respecto al proceso de procuramiento, no contienen todas las etapas y criterios que sí posee nuestro protocolo. Este protocolo conformado por 9 etapas y 36 criterios permitirá estandarizar la cadena de eventos que llevan a un procuramiento exitoso, conocer las falencias y dificultades que surgen en cada etapa, definir las necesidades de capacitaciones específicas, como en Solicitud de donación poder contar con un protocolo para realizar entrevista de STUV, disminuyendo la negativa familiar y facilitando la mejora continua en los distintos procesos de los actuales y futuros coordinadores locales.

Fortaleza de este trabajo, contribuir con una propuesta de protocolo de estandarización y gestión de calidad para el proceso de procuramiento de órganos, desarrollado por expertos nacionales $y$ validado localmente con enfermeros coordinadores de procuramiento de centros clínicos de alta complejidad, lo que contribuirá en la gestión eficaz del proceso en nuestro país

Esperamos que este protocolo se constituya en un referente a nivel nacional, estandarizando el proceso, lo que facilitará el control de gestión de calidad en las diferentes etapas, evidenciando los nodos críticos, para ser intervenidos mejorando de esta forma la calidad del proceso, permitiendo generar indicadores de calidad en un protocolo normado y así poder aumentar las tasas de donantes efectivos.

\section{Referencias}

1. Ojo AO, Hanson JA, Meier-Kriesche H, Okechukwu $\mathrm{CN}$, Wolfe RA, Leichtman $\mathrm{AB}$, et al. Survival in reci- pients of marginal cadaveric donor kidneys compared with other recipients and wait-listed transplant candidates. J Am Soc Nephrol 2001; 12 (3): 589-97.

2. Dueñas JM. Protocolos clínicos de actuación ante el proceso de donación y extracción de órganos y tejidos para trasplante en donación en muerte encefálica. Cuadernos de medicina forense 2015; 21 (1-2): 34-42.

3. Palacios JM. Procuramiento de Órganos: el modelo chileno. Rev Chil Cirug 2002; 54 (6): 575-8.

4. Organización Nacional de Trasplantes (ONT). Newsletter Transplant International figures on donation and transplantation 2016. 2017; 22: 1-72. Disponible en: http://www.ont.es/publicaciones/Documents/NEWSLETTER\%202017_baja\%20(2).pdf [Consultado el 11 de abril de 2018].

5. Organización Nacional de Trasplantes, [Internet] España Disponible en: http://www.ont.es/internacional/Paginas/default.aspx [Consultado el 11 de abril de 2018].

6. Manual de Donación de Órganos para Profesionales Sanitarios [en Internet] Enf. Purificación Gómez Marinero, Coordinador de trasplantes. Dr. Carlos de Santiago Guervós, Coordinador de trasplantes. Hospital General Universitario de Alicante. Disponible en: http://www. bibliotecadigitalcecova.com/contenido/revistas/cat7/ pdf/manual_1.pdf [Consultado el 11 de abril de 2018].

7. El Modelo Español de Donación y Trasplantes [en Internet] disponible en : http://www.ont.es/publicaciones/ Documents/modeloespanol.pdf [Consultado el 11 de abril de 2018].

8. Documento de respaldo al flujograma de procuramiento de órganos y tejidos [en internet] Disponible http:// www.sscoquimbo.cl/doc/documentos/rrhh/15-01-2010/ Doc_difusion.pdf [Consultado el 11 de abril de 2018].

9. Orientaciones técnicas "Proceso de Procuramiento de Órganos y Tejidos para Trasplante" [en Internet] Coordinadora Nacional de Procuramiento y Trasplante de Órganos y Tejidos División de Gestión de Redes Asistenciales Disponible en :http://www.sscoquimbo.cl/ doc/documentos/gestion/19-05 2014/Orientaciones_Organos_y_Tejidos [Consultado el 11 de abril de 2018].

10. Ministerio de Salud de Chile. Datos donantes de órganos y receptores año 2017. [en Internet]. http://www.minsal. cl/wp-content/uploads/2018/01/Datos-P\%C3\%A1gina-Enero-Diciembre-2017.pdf [Consultado el 11 de abril de 2018].

11. Instituto de Salud Pública de Chile. [Internet] Trasplante de órganos y lista de espera por mes año 2016. Disponible en ; http://www.ispch.cl/sites/default/files/ Registro_nacional_de_trasplante_de_organos_informe_a\%C3\%B1o_2016.pdf [Consultado el 11 de abril de 2018]. 
12. Benavente D, Charles F, Orozco R. Detección y manejo médico del donante fallecido en Muerte encefálica. Revista Médica Clínica Las Condes 2010; 21 (2): 166-77.

13. Bugedo G, Bravo S, Romero C, Castro R. Manejo del potencial donante cadáver. Rev Med Chile 2014; 14(12): 1584-93.

14. Hermosilla González PA, Rocha Ortiz S, Rodríguez Vidal M. Cuidados de enfermería en las etapas del proceso donación-trasplante en Chile, aplicado a un caso clínico. Enfermería Nefrológica 2012; 15 (3): 211-7.

15. Rojas JL. Necesidad de órganos en Chile: ¿Qué hacer para progresar? Revista Médica Clínica Las Condes
2010; 21 (2): 152-9.

16. Avilés L, Rivera M, Catoni M. Donar, un cambio de vida: comprender la experiencia de familiares que aceptaron la donación de órganos. Rev Med Chile 2014; 142 (6): 702-6.

17. Rojas JL, Cortés E. Selección y manejo del potencial donante de órganos. Rev Hosp Clin Univ Chile 2007; 18 (4): 281-94.

18. Varela-Ruiz M, Díaz-Bravo L, García-Durán R. Descripción y usos del método Delphi en investigaciones del área de la salud. Investigación en educación médica. 2012; 1 (2): 90-5. 